\title{
Definite Noun Phrases and the Semantics of Discourse
}

\author{
Manfred Pinkal \\ c/o Fraunhofer-Jnstitut IAO \\ Holzgartenstrasse 17, \\ D 7000 Stuttgart 1 \\ and \\ Institut für Linguistik \\ Universität Stuttgart
}

\section{Introduction}

Discourse Representation Theory (DRT), developed by Hans Kamp several years ago (Kamp 1981), belongs, together with Irene Heims narrowly related File Change Semantics (Heim 1982) and Situation Semantics (Barwise/Perry 1983), to a group of theoretical approaches which in the early Eighties introduced a dynamic, context-oriented perspective into the semantics of natural language. This recent development in theoretical semantics indicates a shift of interest towards topics that have been familiar in natural language processing research for the last decade: among others, the interpretation of new utterances with respect to a given context, and integration of the utterance information into that context; the step-by-step construction of representations for larger pieces of discourse; the investigation of text coherence phenomena; and the description of referential processes.

The core of DRT (and File Change Semantics) is the treatment of indefinite noun phrases as reference estabiishing terms (as opposed to their standard truth-conditional quantifier analysis, but in accordance with the treatmant of indefinites in NLP research) and definite noun phrases (pronouns as well as full NPs) as anaphoric expressions. It is one of the theoretically most appealing features of these theories that they provide simple unified accounts for all indefinites, and for all definites, respectively. This theoretical simplicity stands however in sharp contrast to the complexity of the process of etablishing reference observed in NLP research, and the variety of phenomena and linguistic levels involved. On the one hand, this contrast is quite natural: As a semantically motivated theory, DRT should not be expected to incorporate every detail of inferencing necessary to come up with an interpretation for a specific utterance in a given context; it can better be thought of as an interface relating theoretical, truth-conditional semantics and the genuinely pragmatic work of text understanding. On the other hand, if DRT is seriously intended to bridge the gap between theoretical linguistics and the NLP approach, it should take into consideration as many factual restrictions on NP reference, and distinctions among subtypes of referential expressions, as is possible in a systematic and descriptive way. Several extensions of the standard system are at work, e.g. for the treatment of plural and temporal anaphora. Little, however, has yet been done to arrive at a closer view of the analysis of (singular) definite noun phrases, once the basic concepts had been established. The only attempt I know about is by Kamp himself, described in Kamp (1983), an unpublished fragment.

In this talk I will first give a short overview of the basic DRT system, and sketch Kamp's proposal for the treatment of definite noun phrases. Then I will indicate how the basic reference establishing function and the 'side-effects' of different types of definite NPs can be described in more detail. In doing this, I will refer to the work about anaphora done in the NLP area (esp. by
Barbara Grosz, Candy Sidner, and Bonnie Webber), integrating some of their assumptions into the DRT framework, and critically commenting on some others.

\section{The Framework of Discourse Representation Theory}

To illustrate the basic principles of DRT, let us look at the following two-sentence text:

(1) (a) John owns a book.

(b) He reads it.

In an utterance of ( 1 a), two discourse referents (DRs) are introduced by the NPs John and the book, and several data concerning these referents are communicated. These facts about (1 a) are represented by the 'discourse representation structure'(DRS) K1 under (2 a), which consists of a universe of discourse $\left(U_{K}\right)$ as well as a set of conditions $\left(\mathrm{CON}_{K}\right)$ on the members of $U_{K}$.

(2) K1:

$$
\left[\begin{array}{l}
x y \\
\text { John }(x) \\
\text { xowns y } \\
\text { book }(y)
\end{array}\right.
$$

DRSes are built up by DRS construction rules, which operate on a straightforward phrase-structure analysis of the sentence, and are sensitive to the context of utterance. DRSes for texts ate construed by sequentially analysing sentence by sentence, referring to the DRS built up so far as the context of utterance, and extending this DRS by the discourse referents and conditions emerging from the application of the construction rules to the sentence under consideration. Sentence $(1 \mathrm{~b})$ requires application of a very simple context-sensitive construction rule, the rule for anaphoric pronouns, which can roughly be formulated as (3):

(3) (i) Add a new $D R \times$ to $U_{\mathcal{K}}$ ( $K$ being the current $D R S$ ).

(ii) Replace the anaphoric pronoun in the (syntactic description of) the constituent under consideration by $x$.

(iii) Add $\mathrm{x}=\mathrm{y}$ as a new condition, for some DR $\mathrm{y}$ already contained in $U_{K}$

The crucial part of this rule is clause (iii) which says that the pronoun should be anaphorically linked to a referent already present in the context of utterance. By applying (3) twice to (1 b), the DRS K1 is extended to K2.

The author is Heisenberg fellow of the Deutsche Forschungsgemeinschaft. 
(4) K2:

\begin{tabular}{|l|}
\hline$x y z$ \\
John $(x)$ \\
$x$ owns $y$ \\
book $(y)$ \\
$x=z$ \\
$x$ reads $u$ \\
$u=y$
\end{tabular}

The step-by-step construction of a DRS for a given text is the first part of its semantic analysis. The analysis is completed by embedding the DRS representing the text information into a conventional first-order model structure. A proper embedding is a function from $\bigcup_{K}$ to the model universe, assigning real-world objects to DRs in a way that all conditions of the DRS are satisfied. A text is true if a proper embedding for its DRS exists. Thus, the embedding provides a truth-conditional background for the DRS formalism. E.g., it indirectly provides the indefinite NP a book in (1 a), the function of which is described as introduction of a new referent on the DRS level, with its usual existential interpretation.

Conditionals, universal quantifiers, negation require a DRS analysis in terms of complex conditions on sub-DRSes. Sentence (5), e.g. , is represented by the DRS (6), where the left-hand box is the sub-DRS representing the antecedent, and the right-hand box represents the consequent of (5).

(5) If John owns a book, he reads it.

(6)

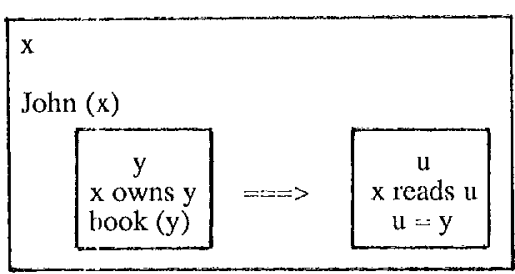

Universal sentences like (7) are represented by the same type of complex DRSes.

\section{(7) Every man who owns a book reads it.}

The embedding rule for $\Rightarrow>$ - conditions requires, roughly speaking, that every proper embedding for the antecedent sub-DRS can be properly extended to the consequent sub-DRS. By this, the indefinite NP a book in (5), which on the DRS-level has identical function with $a$ book in (1 a), i.e. DR introduction, is correctly interpreted as a universal quantifier.

The internal structure of DRSes allows one to formulate an important restriction on the use of anaphoric expressions: DRs introduced in sub-DRSes must not be anaphorically accessed from outside. This restriction excludes a continuation of (5) or (7) by a sentence like (8):

(8) It has 200 pages.

Accessible for anaphoric linking in a DRS $\mathrm{K}$ is $\mathrm{U}_{K}$ itself, the universe of all superordinate DRSes, and, possibly, the universe of the antecedent DRS (if $\mathrm{K}$ represents a consequent of a conditional), but nothing else. This restriction seems to be more or less valid for all kinds of anaphoric expressions; I will not discuss problematic cases here (cf., however, Sells 1985). Instead, I will look at the way Kamp further differentiates between different types of anaphoric noun phrases.

\section{Definite Noun Phrases in DRT}

In his 1983 paper, Kamp distinguishes between four kinds of definite noun phrases:

(i) pronouns (personal and possessive),

(ii) complex demonstratives (this woman, that man who wore a grey coat); bare demonstratives, which have a rather restricted distribution in English, are left out of consideration by Kamp as well as in this paper),

(iii) definite descriptions (the book, the professor who teaches a course on semantics)

(iv) functional definite descriptions (the king of Spain, the author of Waverley), which make up a seperate class of definite NPs and allow elliptic use (the king, the author).

For each of these classes, Kamp states a distinction between a deictic and an anaphoric use, and thus ends up with eight different types of definite noun phrases. The distinction drawn by Kamp between deixis and anaphora is the traditional one: Anaphoric uses of definite NPs refer to an antecedent introduced by the previous discourse; deictic uses of definite NPs refer to an object which is physically present in the situation of utterances, the identification being typically supported by a demonstrative gesture.

Anaphora is interpreted in the way that the new discourse referent introduced for the anaphoric expression is linked by equation to an appropriate discourse referent already contained in the DRS, as was shown above for the pronoun case. DRs introduced for deictically used NPs are anchored to an individual of the real-world model, an anchor being an ordered pair $<x, a>$ consisting of a discourse referent $x$ and an object a. This basic construction schema for deictic expressions in DRT is not very explanatory; also, deixis is of minor importance to natural language systerns (as long as no graphic component is involved). Therefore, I will not go into details of Kamp's description here, but only indicate in a few words the interpretations assigned to the four classes of deictically used NPs.

Deictically used pronouns $(\alpha)$ are anchored to "the object demonstrated by the deictic act accompanying the utterance of $\mathcal{\alpha} "$ (p. 57). Deictically used demonstratives this/that $\alpha$ and deictically (or: referentially) used definite descriptions the $\alpha$ refer to "the unique satisfier[of the common-noun phrase $\alpha$ ] among the objects in the range of the deictic act accompanying the use of the demonstrative" (p. 57). Deictically used elliptic functional descriptions the $\alpha$ are interpreted accordingly; however, the object singled out by the deictic act, or uniquely given by the situation of utterance, does not serve as the referent of the description directly, but instead as the argument of the functional common noun $\alpha$, thus providing a new referent. I will come back to the general problem of the deixis/ anaphora distinction later. In the following, I will concentrate on the anaphoric uses of definite NPs.

The construction rule for anaphoric pronouns has been described already. An new discourse referent is introduced and linked to one of the accessible old referents in the DRS. For the interpretation of anaphorically used complex demonstratives, the DRS concept is augmented by a Lewis style salience ranking on the set of discourse referents (cf. Lewis 1979). The discourse referent for the demonstrative NP this $\alpha$ or that $\alpha$ is, roughly speaking, linked to the most salient referent already contained in the DRS which satisfies the common-noun phrase $\alpha$. Satisfaction of $\alpha$ by $x$ roughly means that the predication $\alpha(x)$ follows from the data available in $\mathrm{K}$ about $\mathrm{x}$, possibly using additional background knowledge. Kamp assumes that the typical use of demonstratives is contrastive; in general, this $\alpha$ implicates that there are other individuals besides the one referred to which also are $\alpha$. 
Anaphoric definite descriptions, on the contrary, carry kind of a uniqueness presupposition. They may only be properly used in cases where exactly one of the objects relevant in a context satisfies the description. Since additional satisfying objects introduced earlier in discourse may prove to be irrelevant for the appropriate use of the description, Kamp further extends the DRS concept by a selection set which is a subset of the DRS universe. (Selection sets were first employed in Cresswell 1973.) The selection set always contains, roughly speaking, an upper portion of the salience ranking <, possibly all of its members. An anaphorically used definite description is linked to its unique satisfier that is member of the selection set. - Elliptic functional definite descriptions take the most salient discourse referent already contained in the DRS as argument of the functional common noun, and link the functorargument expression to the newly introduced referent.

To sum up, the full formalism employed for the treatment of definite NPs consists of

(i) the DRS $K$ with its universe $U_{K}$ (possibly containing a hierarchy of sub-DRSes and their respective universes)

(ii) a salience ranking $<_{K}$ on $U_{\mathcal{K}}$

(iii) a selection set $S \subseteq U_{\mathcal{K}}^{\mathcal{K}}$, and

(iv) the universe of the real world model needed for the interpretation of deictically used NPs.

There are some open questions connected with these formal tools and their interrelation, e.g. how the salience ranking extends to the universe of sub-DRSes. Since a technically correct answer to these questions seems to be unproblematic, and a really explanatory solution would require a specification of an adjustment algorithm for salience ranking and selection set, which Kamp deliberately excludes from his semantic considerations, I will not go into much detail here. Rather, I will concentrate upon some inadequacies in the basic assumptions underlying Kamp's interpretation of definite noun phrases, which require modifications of the over-all framework in several respects.

The probably most striking inadequacy concerns the relation between pronouns and definite descriptions with respect to their anaphoric range. According to Kamp's account, pronouns can link to all discourse referents already introduced into the current DRS and its super-ordinate DRSes, whereas the range of definite descriptions is limited by the selection set (the anaphoric power of demonstratives is restricted by $<k$, and thus lies somehow in between pronouns and descriptions). Actually, the relation is just the opposite one: Pronouns are highly restricted, whereas the range of definite descriptions is nearly universal, as long as the uniqueness condition is not seriously affected. I will try to give an alternative account of the different NP types in terms of their specific anaphoric behaviour taking results of AI work on anaphora into consideration. I will look at pronouns, definite descriptions, and demonstratives, in this order.

\section{Pronominal Anaphora and the Locality Constraint}

In the standard fragment of DRT, anaphoric linking of pronouns is only restricted by the basic accessibility relation. Some additional restrictions, esp. agreement requirements, can easily be added by a sortal classification of discourse referents (for number agreement, see van Eijck 1983, for semantic gender in English, see Johnson/ Klein 1985). Another kind of semantic constraint on anaphoric linking is indirectly provided by the model-theoretic background of DRT: Anaphoric links which lead to an inconsistent extension of the current DRS, prevent embedding into a model structure and thus induce a reanalysis. The relation of DRT and syntactic constraints on anaphora is discussed in Chierchia/ Rooth (1984).
Another important restriction, however, concerning the suprasentential use of pronominal anaphora, has not been noticed in the literature on discourse semantics: the strict limitation of the backward reach of pronouns. Whereas full NPs can refer to arbitrarily remote antecedents, acceptability of pronominal anaphors becomes worse with each additional sentence uttered between pronoun and antecedent. An easy and straightforward account of this fact seems to be provided by a widely accepted assumption about the relation between pronouns and full definite NPs: pronouns are minimal, naked descriptions; full NPs are kind of extended pronouns. Both NP types make reference to the same salience ranking (disregard Kamp's selection set analysis, for the moment). Full NPs may incorporate lots of information in terms of modifiers of different kinds; this enables them to go arbitrarily far down on the salience scale. Pronouns, on the other hand, bear no discriminating information, and thus have to refer to the very first discourse referent on the scale that meets the consistency and agreement requirements. Each intervening object introduced in discourse prevents a linking to a more remote referent .

Persuasive though it is, the explanation does not meet the phenomena. Look at the following example:

(9) (a) Yesterday afternoon, I went shopping

(b) I bought an interesting book on semantics

(c) Afterwards, I met some friends.

(d) In the evening, I went dancing with Mary, and we came home late.

(e) It was midnight when I started reading it.

The pronoun it in $(9$ e) sounds definitely peculiar, and things don't become much better if the intervening text is reduced in length. However, the book of ( $9 \mathrm{~b})$ is the only inanimate referent explicitly introduced so far, and therefore should be on top of the salience ranking, the book (as well as that book) instead of it in (9 e) is perfectly fine, although the common noun does not provide additional information in the situation under consideration, particularly if one takes into account that the choice is restricted to readable objects. Furthermore, look at (10), as an alternative to (9 e).

(10) It was after mid-night when I started reading

Here, the hearer will easily infer from the previous discourse that the omitted object is the book from $(9 \mathrm{~b})$. It does not matter for my argument that this inference is just default and can be explicitly denied by the next utterance: The book is the most salient candidate, and an easily recoverable one, too. So, the oddity of the pronoun use in (9) must have a different reason. The only reason I can see is that the supposed antecendent is simply too old. Different from full noun phrases, anaphoric pronouns must find their antecedent in the local linguistic context, roughly speaking, in the sentence in which they occur, or in the immediately preceding sentence, independently of what the over-all salience ranking looks like. If a referent is not rementioned, it soon drops out of the current universe'for pronominal anaphora.

To integrate the locality constraint into DRT, several changes are in order. A concept of current universe $\mathrm{CU}_{k}$ must be employed, $\mathrm{CU}_{K}$ being a distinguished subpart of the universe of discourse and consisting of a current-sentence position $\left(\mathrm{CU}_{0, \kappa}\right)$ and a lastsentence position $\left(\mathrm{CU}_{1, k}\right)$.

$$
\text { (11) } \begin{aligned}
\mathrm{CU}_{K} & \subseteq \mathrm{U}_{K} \\
\mathrm{CU}_{K} & =\mathrm{CU}_{o, K} \cup \mathrm{CU}_{1, K}
\end{aligned}
$$

New referents are introduced as members of $\mathrm{CU}$. The syntactic description is completed by an end-of-sentence marker, and the following construction rule for this marker is added. 
(12) Set $\mathrm{CU}_{1, k}$ to $\mathrm{CU}, \mathrm{K}$ Set $\mathrm{CU}_{0, \kappa}$ to $\phi$

Note, that only the topmost DRS is affected by this rule. For subordinate DRSes no current universe management is necessary because their discourse referents become inaccessible before they get old. Finally, the construction rule for anaphoric pronouns needs a slight reformulation; (3) (iii) has to be replaced by:

\section{(13) Add $\mathrm{x}=\mathrm{y}$ to $\mathrm{K}$ for some $\mathrm{y} \in \mathrm{CU}_{\mathcal{K}}$}

This is only a first attempt to fix the local range of pronominal anaphora. Much would have to be said to the outer boundary of the current universe (the end-of-sentence marker being only a crude approximation to an adequate concept of local text structure) as well as to its internal structure, to which I will come back later. However, there are several basic objections and apparent counterexamples to the locality constraint on pronominal anaphora, which I will shortly comment on, now. First of all, there is the objection that a hearer of (9) is able to assign the correct reference to the pronoun despite its oddity. This statement is of no different status than the observation that hearers are able to make scnse out of ungrammatical utterances (although, admittedly, acceptability statements are usually clearer for syntactic than for pragmatic data).

Firther, (rosz et al. (1983) observe that in task-oriented dialogues the pronoun it may refer back to the topic of primary interest, no matter when it has been introduced. This case seems to be related to quasi-deictic uses of $i t$, e.g., if (14) is uttered as the first sentence in a conversation between people waiting at the bus stop:

\section{(14) It will arrive in a moment.}

The current universe can be 'invaded' by objects of central importance in a situation; this is, however, a marked case applying only in situations clearly dominated by one object, and must be distinguished from ordinary salience considerations. In the Grosz cases, there is no direct connection between pronoun and supposed antecedent. The 'chain of reference' takes its way through the nonlinguistic situation. Evidence for this assumption is provided by languages with syntactic gender like German: there is strict syntactic gender agreement between anaphor and (local) antecedent; no such agreement holds in the cases described by Grosz.

Third, the pronouns he and she have distinctly wider range than it:. This seems to be evidence for the naked description interpretation of pronouns, and counter-evidence against the locality constraint: since he and she carry more information, they allow to go further down on the salience ranking. However, er and sie as the masculine and feminine pronouns in German have no further reach than es (and $i t$ in English), as long as they link to inanimate objects. Pronouns referring to humans on the other hand, can be used more freely, they also bear contrastive stress, and allow unmarked deictic uses. The behaviour of these pronouns suggests that they go with full NPs, whereas inanimate pronouns constitute a seperate class. It does not affect the locality statement for the latter.

\section{Definite Descriptions}

In contrast to pronouns, definite descriptions are not sensitive to the concept of current universe. They may anaphorically link to arbitrarily remote antecedents. Moreover, where pronouns (at least the inanimate cases) are constrained to anaphoric use, descriptions apply freely to objects introduced in discourse, present in the physical environment, or available through the common background of the discourse participants. The most salient object meeting the description is selected as the referent, independently of its offspring. The uniqueness condition, which Kamp models with selection sets, should better be viewed as a very general conversational requirement, i.e. that the intended object of reference must be unambiguously more salient than each other possible candidate. A salience ranking as Kamp proposes for the interpretation of complex demonstratives, seems to be the appropriate means to interpret definite descriptions, on an abstract level. There is no motivation for a distinction between a deictic and an anaphoric use (a distinction which anyway becomes problematic for the common background containing all kinds of old information), and, consequently, for a sharp distinction between explicitly introduced discourse referents, and other reference objects. The universe of discourse $U_{\mathcal{K}}$ is a more comprehensive set, containing of course all reference objects explicitly introduced, but not as a distinguished subset. Thus, there will be just one general rule for descriptions the $\alpha$ :

(15) (i) Add a new DR $x$ to $C U_{K}$

(ii) Replace the $\alpha$ in the constituent under consideration by $x$.

(iii) Add $x=y$ to $K$, where $y$ is the most salient referent in $U$ that satiesfies the description $\alpha$.

I will not comment on some questions here that are crucial for practical discourse processing: How is the set of additional reference objects cstablished? How is knowledge about these additional reference objects represented? And most important: What is the mechanism underlying the constitution and the modification of the salience ranking? Kamp suggests that background information be stored in some DRS-like format (Kamp 1985). As far as the salience of reference objects for descriptions is concerned, it seems that more complex, script-like structures, containing large amounts of common-sense information about spccific types of situation, and guiding speakers' expectations about the continuation of the discourse, play a crucial role (cf. Grosz' 1977 work on focus spaces). 'To sum up, definite descriptions are that type of NP showing the least degree of linguistic pre-structuring in their anaphoric behaviour, and the strongest dependence on world knowledge.

This everything holds for plain (non-functional) definite descriptions. Functional definite descriptions in their elliptic use also do not make a difference between linguistic and non-linguistic context. As I think, however, it is not the best way to describe them as functions in the mathematical sense operating on DRs which have been isolated throtgh salience considerations, as in the case above. They rather seem to express functions in the sense of the role concept specified in Situation Semantics. I will not go into more detail, here.

\section{Complex Demonstratives and Pronouns as Focus Indicators}

The analysis of definite descriptions proposed in the last section is based on the same concept of salience ranking that Kamp employs for complex demonstratives. Actually, reference seems to be established for both kinds of noun phrases in basically the same way: that $\alpha$, as well as the $\alpha$, is sensitive neither to the locality constraint nor to the distinction between linguistic and nonlinguistic context. I would maintain the salience ranking analysis also for demonstratives. (I will comment on this $\alpha$ in a moment.) So, what makes up the difference between descriptions and demonstratives, which undoubtably exists?

Kamp notes that a demonstrative this/that $\alpha$ carries an implicature that there is more than one object satisfying $\alpha$, and in connection with this Qbservation argues that demonstratives have contrastive function. Either statement meets a characteristic feature of demonstratives. Both, non-uriqueness and contrast, should however be regarded and can be explained in a wider connection, none of them being the essential property of demonstratives. 
Kamp gives as evidence for his non-uniqueness assumption examples like that pope, which is odd or at least highly marked. However, there are clear cases of descriptions with a single satisfying object which combine easily with the demonstrative that, e.g. that small planet beyond Uranus, or that woman who sold me thebook on Situation Semantics. On the other hand, that father is as odd as that pope, although nothing is uncommon about a situation where several fathers are present. These facts suggest the following explanation: The article the has two distinct uses - plain descriptive and functional descriptive use, as Kamp calls it whereas that is restricted to the former. Thus, the use of that forces a predicative rather than a functional reading of the common noun. This explains the markedness of cases with strongly preferred functional reading, like pope and father. (Sun and universe belong to the same group, which can be seen $e . g$. from the distribution of preposition-article contraction indicating 'functional' use in German.)

Actually, there are cases where a strong non-uniqueness presupposition or implicature is carried by a demonstrative phrase, i.e., cases with contrastive stress on the demonstrative pronoun and unstressed common-noun phrase as in that professor or that professor with the old Mercedes. However, the presupposition goes with the stress here, and is not a specific property of the demonstrative. In the complementary case - stress on (part of) the common-noun phrase (and optionally on the determiner, in addition) - the presupposition fades away. That professor with the old Mercedes implicates only that there is some other professor involved in the situation. That professor and, finally, that professor with the old Mercedes without any stress marking, seem to implicate nothing than the very general fact that there are other objects under consideration. This implicature follows from what I consider in accordance with Sidner (1984) as the basic property of demonstrative phrases. The main function of demonstratives is that they direct the hearer's focus of attention to a new reference object that was not in focus before. There is a lot of clear evidence for this; I just give the following pair of examples for illustration.

(16) Do you remember that professor with the old Mercedes?

(17) Do you remember the professor with that old Mercedes?

In (16) it is the professor, in (17) it is the old Mercedes, which is understood by the hearer as the primary topic for the next utterance (and by this, as the preferred candidate for pronominal anaphora).

Since Kamp explicitly excludes the updating mechanism for the salience ranking from consideration, his analysis cannot model the focus-shifting effect of demonstratives. Also, the extension of the DRS formalism proposed so far in my talk is insufficient. The current universe $\mathrm{CU}_{K}$ contains potential topics, potential referents for personal pronouns, whereas the use of a demonstrative moves a referent definitely into the focus of attention. So let me introduce $\mathrm{CF}_{K} \subseteq \mathrm{CU}_{K}$, standing for the 'current focus of DRS. $\mathrm{K}^{\prime}$ (in addition $\mathrm{CF}_{0, \kappa} \kappa$ and $\mathrm{CF}_{1, k}$ are needed as in the current universe case). The pair CU/CF corresponds to Grosz' distinction between forward and backward thematic center, and the opposition of potential and confirmed focus in Sidner's analysis. The rule for that $\alpha$ is given in (18).

(18) (i) Add a new DR to $\mathrm{CF}_{\kappa}$

(ii) as (3) (ii) or (15) (ii)

(iii) Add $\mathrm{x}=\mathrm{y}$ to $\mathrm{K}$ where $\mathrm{y} \notin \mathrm{CF}_{K}$ and $\mathrm{y}$ is the most salient satiesfier of $\alpha$ in $U_{K}$.

The corresponding rule for this $\alpha$ is like (18), except the additional requirement that the antecedent must have been uttered in the immediate pre-context. It is tempting to apply the already introduced concept of current universe to make the closeness condition more precise. However, this has a yet more restricted anaphoric range, which seems to depend on proximity in the uttered string, in a very basic sense.

(19) I asked an old man and a young woman.

Reference to the second conjunct in (19) by this woman is much better than reference to the first conjunct by this man. In its prototypical deictic use, this is a case of local deixis (cf. Levinson 1983), and this property seems to be inherited in its anaphoric (or better: text-deictic) use.

The use of demonstratives is only one way of explicit focus indication. In addition, prosodic means (stress, intonation) and specific syntactic constructions (topicalization, cleft and pseudocleft constructions) are available. I will not comment on these here; Sidner (1984) gives a rather comprehensive overview of focus indicators in English. There is one case of focus indicators, however, $\mathbb{I}$ have to deal with, since it concerns a class of NPs for which I gave already a preliminary analysis. Personal pronouns are in two ways connected with the focus concept. On the one hand the choice of an antecedent is dependent of the current focus. Second, by pronominal reference to a $D R$ in the current universe this DR is confirmed as the actual focus. The modified construction rule for pronouns is given in (20).

(20) (i) Add a new discourse referent to $\mathrm{CF}_{K}$.

(ii) $=$ (3) (ii)

(iii) Add $\mathrm{x}=\mathrm{y}$ to $\mathrm{K}$ where $\mathrm{y} \in \mathrm{CF}_{K}$, if $\mathrm{CF}_{K} \not \varnothing \varnothing$ (and $\mathrm{CF}_{K}$ is not yet exhausted by other anaphoric references); $\in \in \mathrm{CU}_{\mathcal{K}}$ else.

Demonstratives refer to a DR not yet confirmed as focus and move it into CF. Pronouns refer to members of CU, preferably to explicitly focussed DRs, and keep them in CF. These facts allow some predictions about the distribution of demonstratives and pronouns in adjacent sentences. The most obvious one is that two demonstratives in immediately succeeding sentences should not be anaphorically linked to each other (violations are possible, but have a stylistic or connotational effect). Further, (18) together with (20) predicts that a pronoun is a bad antecedent for a demonstrative. The following sequence looks like a counter-example.

(21) (a) Last week, I bought a new dish-washer.

(b) It works really fine.

(c) I had seen this dish-washer in a commercial

At a closer look, however, it turns out that the focus shifts from the physical object (my dish-washer) to a generic object (a certain brand of dish-washer) between $(21 \mathrm{~b})$ and $(21 \mathrm{c})$. This effect (which is systematically used by speakers) seems to be caused by the fact that a direct anaphoric reading is excluded by (18) and (20): a confirmation rather than counter-evidence for the proposed analysis. (There is, however, a serious problem for DRT contained in (21): Where does the generic reference for this dish-washer in (21 c) come from?)

Finally, Grosz et al. (1983) discuss a rule for the cooccurence of pronouns in adjacent sentences, stating that "If the $\mathrm{Cb}$ ['backwardlooking center'] of the current utterance is the same as the $\mathrm{Cb}$ of the previous utterance, a pronoun should be used." The rulc predicts the oddity of examples like the following:

(22) (a) $\mathrm{He}_{i}$ called up Mike ${ }_{j}$ yesterday.

(b) $\mathrm{He}_{j}$ was annoyed by John $i$ 's call.

The 'backward-looking center' concept roughly corresponds to the current focus of my proposal. The main difference is that Grosz et al. assume the existence of only one centered referent at a time (exceptions due to the ambiguity of NPs between referential and intensional readings being of no importance here), whereas the 
cardinality of $\mathrm{CF}$ is unlimited in principle. Thus, the rule cited above turns out to be a special consequence of the more general pronoun construction rule (20): All CF members have to be consumed first, before pronominal reference can be made to DRs that are not explicitely focussed. There is one difficulty arising from the procedural formulation of DRT rules, which presupposes left-to-right processing: This would restrict access to CF to the leftmost pronoun(s), which means in the above example that the subject he of (b) must have index $\mathrm{i}$ irrespective of whether another pronoun follows. By a declarative formulation of DRT, this undesirable effect can be avoided.

\section{Concluding Remarks}

Kamp (1983) treats the different types of definite NPs in the DRT framework by integrating conventional model-theoretic analyses of descriptions and demonstratives. I have argued in this paper that a more adequate analysis is possible, which utilizes concepts and results from NLP research. It is one of the advantages of DRT over standard model-theoretic semantics that it allows a straightforward integration of these concepts. In order to specify the functions of different definite NP types, I have proposed two extensions of the basic DRT formalism: First, the locality constraint, which is formally represented by $\mathrm{CU}$ and allows to describe the difference in anaphoric behaviour between pronouns on the one hand and descriptions and demonstratives on the other hand (which difference I would tend to consider as more basic and categorical than is usually done in NPL work on anaphora). Second, focus indication explains the functional difference between definite descriptions and demonstrative phrases as well as constraints on the cooccurence of co-specifying NPs. It is represented by $\mathrm{CF}$.

Let me conclude with a remark on the relation between $\mathrm{U}, \mathrm{CU}$, and $\mathrm{CF}$. Since $\mathrm{CF}_{K} \subseteq \mathrm{Ci}_{K} \subseteq \mathrm{U}_{K}$, since $\mathrm{CF}_{K}$ as well as $\mathrm{CU}_{K}$ are sets the members of which differ in therr readiness to serve as antecedents for anaphoric pronouns, one might think of considering $\mathrm{CF}_{K}$ and $\mathrm{CU}_{K}$ as different top portions of the salience ranking on $U_{K}$, and perhaps doing away with $\mathrm{CF}_{K}$ altogether, describing the focus indicating function of demonstratives and pronouns by modification of the salience ranking. There are several reasonis why this approach cannot lead to a satisfactory solution. One of them is that $\mathrm{CF}$ members as "confirmed focus elements have in epistemic status which qualitatively distinguishes them from all other DRs in the universe of discourse, and which would be only insufficiently represented by making them top members of the salience ranking. Also, it would be inadequate to refer to the same salience ranking for global and local anaphora, for $U$ and $C U$ : Preferences for pronominal anaphora depend essentially on syntactic (surface and functional) criteria, which don't play a role for definite descriptions. Finally, a linear salience ranking would be a much too simple formalism to represent preference data for local, pronominal anaphora. Parallelism plays an important role on different levels: Pronominal surface subjects tend to anaphorically refer to surface subjects; pronouns refer more casily to antecedents that occupy the same thematic position, a fact that led Sidner to split the focus concept into 'actor focus'and 'discourse focus'. Thus, a discourse may be pervaded by several more or less independent chains of anaphoric relations. This aspect of anaphoric text structure cannot be straightforwardly integrated into the DRT framework. A more elaborate syntactic foundation of discourse semantics is required.

\section{References}

Barwise, J./ Perry, J. (1983), Situations and Attitudes. Cambridge, Mass.: MIT Press

Chierchia, G./ Rooth, M. (1984), Configurational Notions in Discourse Representation Theory. In: NELS 14,GLSA, University of Massachusetts, Amherst, Mass., p. 49-63

Cresswell, M.J. (1973), Logics and Languages. London: Methuen

van Eijck, $J .(1983)$, Discourse representation Theory and Plurality. In: ter Meulen, A. (ed.), Studies in Model-theoretic Semantics. GRASS 1 Dordrecht: Foris

Grosz, B. (1977), The Representation and Use of Focus in a System for Understanding Dialogs. SRI-Report, Menlo Park CA

Grosz, B./ Joshi, A./ Weinstein, S. (1983), Providing a Unified account of Definite Noun Phrases in Discourse. SRI Technical Note, Menlo Park, CA

Heim, I. (1982), The Semantics of Definite and Indefinite Noun Phrases. Diss. Amherst, Mass.

Johnson, M./ Klein, E. (1985), A Declarative Formulation of DRS Theory. Notes from the ASL Talk, Stanford, CA

Kamp, H. (1981), A Theory of Truth and Semantic Representation. In: Groenendijk et al. (eds.), Formal Methods in the Study of Language. Amsterdam

Kamp, H. (1983), SID without Time or questions. Ms. Stanford, CA

Kamp, H. (1985), Context, Thought, and Communication. In: Proc. of the Mecting of the Aristotelian Society

Levinson,S. (1983), Pragmatics. Cambridge UP

Lewis, D. (1979), Score-keeping in a Language Game. In: Büuerle et al. (eds.), Semantics from Different Points of View, Berlin: Springer

Sells, $P$. (1985), Restrictive and Non-restrictive Modification. CSLI-Report, Stanford, CA

Sidner, C. (1980), Focus for Interpretation of Pronouns. Ms Cambridge, Mass.

Sidner, C. (1984), Focussing in the Comprehension of Definite Anaphora. In: Brady, M./ Berwick, R. (eds.), Computational Models of Discourse. Cambridge, Mass.: MIT Press

Webber, B. (1984), So what can we talk about now?. In: Brady, M. /Berwick, R. (eds.), Computational Models of Discourse, Cambridge, Mass.: MIT Press 